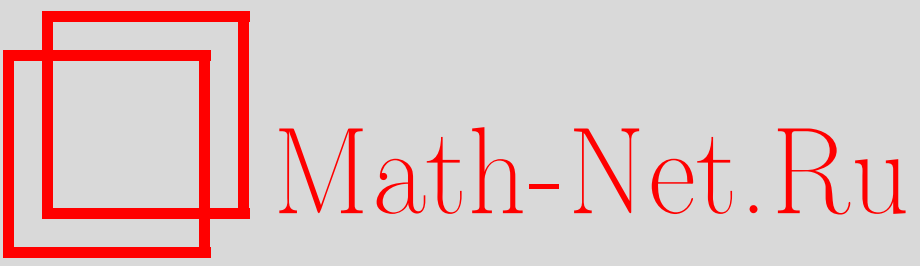

О. А. Фролова, Математическое моделирование предельного напряженного состояния тонкого цилиндрического слоя из сжимаемого сыпучего материала, Итоги науки и техн. Сер. Соврем. мат. и ее прил. Темат. обз., 2021, том 192, 125-130

DOI: https://doi.org/10.36535/0233-6723-2021-192-125-130

Использование Общероссийского математического портала Math-Net.Ru подразумевает, что вы прочитали и согласны с пользовательским соглашением

http://www.mathnet.ru/rus/agreement

Параметры загрузки:

IP : 54.147 .182 .235

26 апреля 2023 г., 10:09:54 


\title{
МАТЕМАТИЧЕСКОЕ МОДЕЛИРОВАНИЕ ПРЕДЕЛЬНОГО НАПРЯЖЕННОГО СОСТОЯНИЯ ТОНКОГО ЦИЛИНДРИЧЕСКОГО СЛОЯ ИЗ СЖИМАЕМОГО СЫПУЧЕГО МАТЕРИАЛА
}

\author{
(c) 2021 г. $\quad$ О. А. ФРОлОВА
}

\begin{abstract}
АннотАция. Рассмотрена математическая модель предельного напряженного состояния тонкого вертикального цилиндрического слоя, заполненного сжимаемым сыпучим материалом, находящимся под действием вертикальной нагрузки. Получены выражения, определяющие напряженное состояние. Оценена зона пластического деформирования материала в зависимости от коэффициентов трения.
\end{abstract}

Ключевые слова: математическое моделирование, предельное напряженное состояние, сыпучий материал, осесимметричное деформирование.

\section{MATHEMATICAL MODELING \\ OF LIMITING STRESS STATES \\ OF A THIN CYLINDRICAL LAYER \\ OF COMPRESSIBLE BULK MATERIAL}

\author{
(c) 2021 O. A. FROLOVA
}

\begin{abstract}
A mathematical model of limiting stress states of a thin vertical cylindrical layer filled with compressible bulk material under the action of a vertical load is considered. Parameters of the stress state are calculated. The zone of plastic deformation of the material is estimated as a function of the friction coefficients.
\end{abstract}

Keywords and phrases: mathematical modeling, limiting stress state, bulk material, axisymmetric deformation.

AMS Subject Classification: 35Q74

1. Введение. Рассматривается математическая модель полупространства, заполненного связным сыпучим материалом, в цилиндрической системе координат $r, \theta, z$. Предполагается, что внешние нагрузки и смещения обладают осевой симметрией, следовательно деформации и напряжения обладают свойством осесимметричности, т.е. не зависят от угла $\theta$.

Для рассматриваемой математической модели компоненты тензора скорости деформации, учитывающие микроструктуру материала, имеют следующий вид (см. $[1,4,9,10])$ : 




Рис. 1. Условия пластичности: 1 - условие пластичности Кулона-Мизеса, 2 - замкнутое условие пластичности.

$$
\begin{array}{ll}
\varepsilon_{r r}=\frac{\partial U_{r}}{\partial r}+\frac{h^{2}}{6}\left(\frac{1}{r} \frac{\partial^{2} U_{r}}{\partial r^{2}}+\frac{\partial^{3} U_{r}}{\partial r^{3}}+\frac{\partial^{3} U_{r}}{\partial z^{2} \partial r}\right), & \varepsilon_{\theta \theta}=\frac{U_{r}}{r}+\frac{h^{2}}{6}\left(\frac{1}{r} \frac{\partial^{2} U_{r}}{\partial r^{2}}+\frac{1}{r} \frac{\partial^{2} U_{r}}{\partial z^{2}}+\frac{1}{r^{2}} \frac{\partial U_{r}}{\partial r}\right), \\
\varepsilon_{z z}=\frac{\partial U_{z}}{\partial z}+\frac{h^{2}}{6}\left(\frac{\partial^{3} U_{z}}{\partial z^{3}}+\frac{\partial^{3} U_{z}}{\partial r^{2} \partial z}+\frac{1}{r} \frac{\partial^{2} U_{z}}{\partial r \partial z}\right), \quad \varepsilon_{r \theta}=\varepsilon_{\theta z}=0, \\
\varepsilon_{r z}=\frac{1}{2}\left(\frac{\partial U_{r}}{\partial z}+\frac{\partial U_{z}}{\partial r}\right)+\frac{h^{2}}{12}\left(\frac{\partial^{3} U_{r}}{\partial r^{2} \partial z}+\frac{1}{r} \frac{\partial^{2} U_{r}}{\partial r \partial z}+\frac{\partial^{3} U_{r}}{\partial z^{3}}+\frac{\partial^{3} U_{z}}{\partial r^{3}}+\frac{1}{r} \frac{\partial^{2} U_{z}}{\partial r^{2}}+\frac{\partial^{3} U_{z}}{\partial r \partial z^{2}}\right),
\end{array}
$$

где $h$ - параметр микроструктуры.

Математическая модель рассматриваемого напряженного состояния описывается двумя уравнениями равновесия и условием пластичности (см. $[2,3,6-8])$ :

$$
\begin{gathered}
\frac{\partial \sigma_{r r}}{\partial r}+\frac{\partial \sigma_{r z}}{\partial z}+\frac{1}{\sqrt{2}}\left(I_{1 \sigma}\right)_{, z}+\frac{\sigma_{r}-\sigma_{\theta}}{r}=0, \\
\frac{\partial \sigma_{r z}}{\partial r}+\frac{\partial \sigma_{z}}{\partial z}-\frac{1}{\sqrt{2}}\left(I_{1 \sigma}\right)_{, r}+\frac{\sigma_{r z}}{r}-\frac{1}{r \sqrt{2}} I_{1 \sigma}=-\gamma, \\
\Phi=\sigma_{r r}^{2}+\sigma_{\theta \theta}^{2}+\sigma_{z z}^{2}+2 \sigma_{r z}^{2}-\beta I_{1 \sigma}^{2}-2 \alpha Y I_{1 \sigma}-Y^{2}=0,
\end{gathered}
$$

где $\beta=1 / 3+\alpha^{2}-f^{2}, \alpha$ - коэффициент внутреннего трения, $f$ - коэффициент трения качения, $\gamma$ - сила тяжести, $Y$ - сцепление.

Наличие микроструктуры в представительном элементе рассматриваемой модели предполагает возможность объемной пластической деформации за счет сдвига, и тем самым имеет место зависимость предельного касательного напряженного состояния от давления. Таким образом, представляется оправданным вид замкнутого условия пластичности (3) в пространстве главных напряжений. Из условия (3) следует ограничение на определение области существования условия пластичности на оси $I_{1 \sigma}$

$$
f \leqslant\left(\alpha+\frac{Y}{I_{1 \sigma}}\right)
$$

которое позволяет описать множество условий пластичности в плоскости инвариант тензора напряжения (рис. 1). 
На рис. 1 представлено множество условий пластичности: 1 - условие пластичности КулонаМизеса; 2-замкнутое условие пластичности. Условие пластичности в пространстве второго инварианта симметричного тензора напряжений и первого инварианта тензора напряжений представляют собой семейства: гипербол при $\alpha>f$, парабол при $\alpha=f$ или эллипсов при $\alpha<f$. При условии малости коэффициента трения качения $f \approx 0$ условие пластичности (3) переходит в условие пластичности Мизеса-Шлейхера, которое при малом угле внутреннего трения $\alpha \approx 0$ переходит в условие пластичности Мизеса.

2. Построение математической модели напряженного состояния деформирования тонкого цилиндрического слоя. Для рассматриваемой математической модели деформирования цилиндрического слоя из сжимаемого сыпучего материала компоненты перемещений удовлетворяют условиям $U_{r}=0, U_{z}=U_{z}(z)$, что кинематически соответствует деформированию тонкого вертикального цилиндрического стержня под действием вертикальных нагрузок.

С учетом сделанного предположения компоненты тензора скорости деформации (1) примут вид

$$
\varepsilon_{r r}=\varepsilon_{\theta \theta}=\varepsilon_{r z}=0, \quad \varepsilon_{z z}=\frac{\partial U_{z}}{\partial z}+\frac{h^{2}}{6} \frac{\partial^{3} U_{z}}{\partial z^{3}} .
$$

Система уравнений (2)-(3), описывающая математическую модель напряженного состояния, содержит три уравнения и четыре неизвестные, т.е. является незамкнутой. Для замыкания системы уравнений в напряжениях воспользуемся ассоциированным законом пластического течения, который связывает скорость деформации и напряженное состояние (см. $[2,6])$ :

$$
\varepsilon_{i j}=\lambda \frac{\partial \Phi}{\partial \sigma_{i j}}
$$

где $\lambda$ - неопределенный множитель Лагранжа.

Дифференцируя условия пластичности (3) с учетом (4), (5), получим

$$
\begin{gathered}
2 \lambda\left(\sigma_{r r}-\beta I_{1 \sigma}-\alpha Y\right)=0, \\
2 \lambda\left(\sigma_{\theta \theta}-\beta I_{1 \sigma}-\alpha Y\right)=0, \\
2 \lambda\left(\sigma_{z z}-\beta I_{1 \sigma}-\alpha Y\right)=\frac{\partial U_{z}}{\partial z}+\frac{h^{2}}{6} \frac{\partial^{3} U_{z}}{\partial z^{3}}, \\
4 \lambda \sigma_{r z}=0 .
\end{gathered}
$$

Из уравнения (9) следует, что $\sigma_{r z}=0$. Из уравнений (6) и (7) следует, что $\sigma_{r r}=\sigma_{\theta \theta}$.

Так как напряженное состояние зависит только от осевой координаты $z$ и $\sigma_{r r}=\sigma_{\theta \theta}$, то уравнения равновесия (2) и условие пластичности (3) примут вид

$$
\begin{gathered}
\frac{f}{\sqrt{2}}\left(I_{1 \sigma}\right)_{, z}=0, \quad \frac{\partial \sigma_{z}}{\partial z}-\frac{f}{r \sqrt{2}} I_{1 \sigma}=-\gamma, \\
\Phi=2 \sigma_{r r}^{2}+\sigma_{z z}^{2}-\beta I_{1 \sigma}^{2}-2 \alpha Y I_{1 \sigma}-Y^{2}=0 .
\end{gathered}
$$

Из первого уравнения (10) следует, что первый инвариант тензора напряжений постоянен, т.е. $I_{1 \sigma}=C_{1}=$ const; тогда из второго уравнения (10) получим

$$
\frac{\partial \sigma_{z z}}{\partial z}=\frac{f C_{1}}{r \sqrt{2}}-\gamma
$$

Проинтегрировав последнее уравнение, получим выражение для осевой компоненты напряжения:

$$
\sigma_{z z}=\left(\frac{f C_{1}}{r \sqrt{2}}-\gamma\right) z+C_{2}
$$

Предполагается, что к цилиндрическому слою приложена вертикальная нагрузка $p_{0}$, удовлетворяющая начальному напряженному состоянию $\sigma_{z z}^{0}, \sigma_{r r}^{0}, \sigma_{\theta \theta}^{0}$.

На границе $z=0$ из выражения (12) получим $C_{2}=\sigma_{z z}^{0}=-p_{0}$. 
(a)

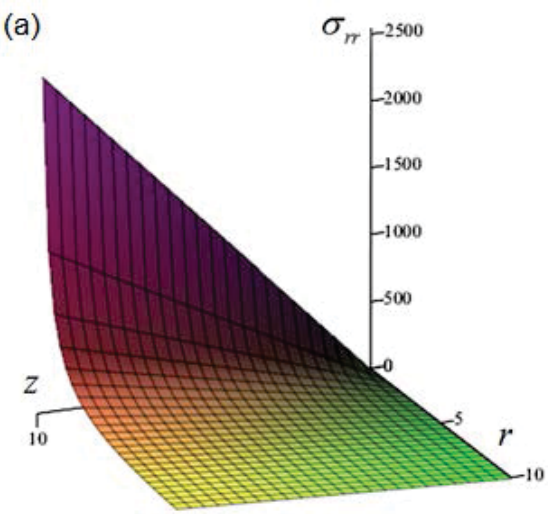

(b)

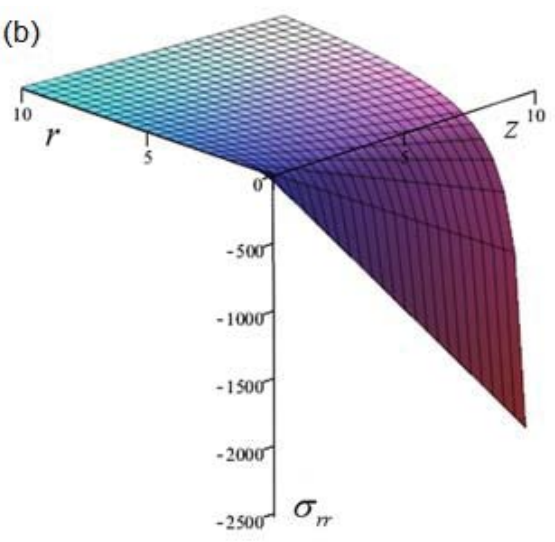

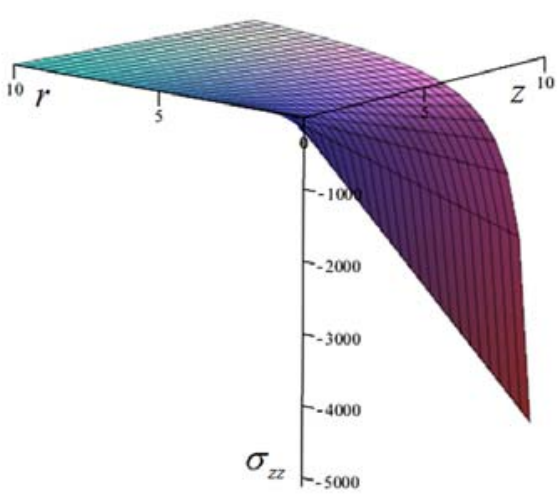

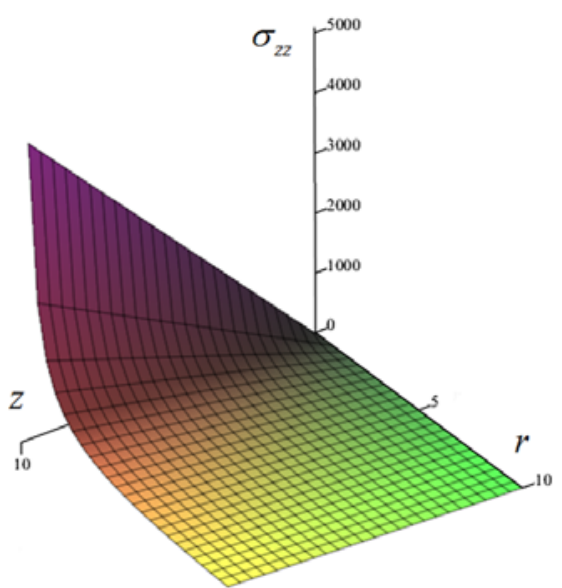

Рис. 2. Напряженное состояние для случаев: (а) сжатия, (b) растяжения.

Первый инвариант напряжений, соответствующий начальному напряженному состоянию, имеет вид

$$
I_{1 \sigma}=C_{1}=\sigma_{r r}^{0}+\sigma_{\theta \theta}^{0}+\sigma_{z z}^{0}=-3 p_{0} .
$$

Выражение (12) с учетом $C_{2}$ примет вид

$$
\sigma_{z z}=\left(\frac{-3 f p_{0}}{r \sqrt{2}}-\gamma\right) z-p_{0}
$$

Так как $I_{1 \sigma}=C_{1}=-3 p_{0}$, то

$$
\sigma_{r r}=\sigma_{\theta \theta}=-p_{0}+\frac{1}{2}\left(\frac{3 f p_{0}}{r \sqrt{2}}+\gamma\right) z .
$$

Уравнения (14), (15) описывают математическую модель напряженного состояния (рис. 2).

3. Оценка зоны пластического деформирования. Рассмотрим условие пластического деформирования материала (11). С учетом выражения первого инварианта тензора напряжений (13) и выражений для компонент напряжения (14)-(15), получим

$$
\Phi=\left(\frac{A z}{r}+\gamma z-2 p_{0}\right)^{2}+2\left(\frac{A z}{r}+\gamma z+p_{0}\right)^{2}-18 \beta p_{0}^{2}+12 \alpha Y p_{0}-2 Y^{2}=0, \quad A=\frac{3 f p_{0}}{\sqrt{2}} .
$$

Решив уравнение (16) относительно $z$, получим выражение для оценки зоны пластического деформирования материала

$$
z= \pm \frac{B r}{A+\gamma r}
$$




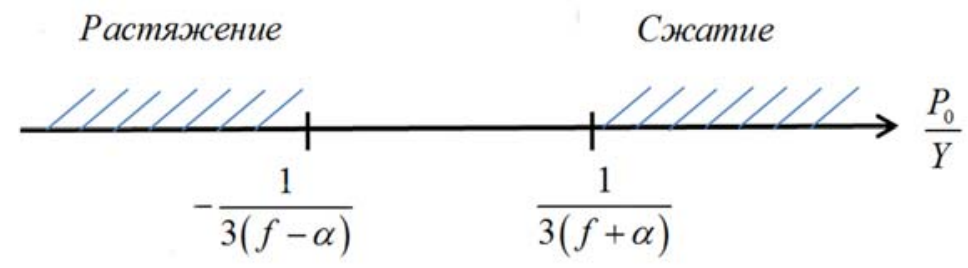

Рис. 3. Зона пластического деформирования материала.

где

$$
B^{2}=6 p_{0}^{2}\left(\alpha^{2}-f^{2}\right)-4 \alpha Y p_{0}+\frac{2}{3} Y^{2}
$$

Рассмотрим выражение для $B$. Разделив его на $Y^{2}$ и введя обозначение $k=p_{0} / Y$, получим

$$
3 k^{2}\left(\alpha^{2}-f^{2}\right)-2 \alpha k+\frac{1}{3} \geqslant 0 .
$$

Взяв в последнем неравенстве знак $=$, получим

$$
k_{1,2}=\frac{\alpha \pm f}{3\left(\alpha^{2}-f^{2}\right)} .
$$

Выражение (17) позволяет оценить зону пластического деформирования материала для случаев сжатия и растяжения.

4. Выводы. Из рис. 2 следует, что напряжения уменьшаются с увеличением радиуса цилиндрического слоя. Имеет место линейная зависимость осевых напряжений вдоль оси $z$ за счет собственного веса. При этом выполняется условие

$$
\sigma_{z z}+p_{0}=-2\left(\sigma_{r r}+p_{0}\right)=-2\left(\sigma_{\theta \theta}+p_{0}\right) .
$$

Полученный вид напряженного состояния соответствует классической технической гипотезе распределения напряжения в грунтах (см. [5]).

При принятых кинематических условиях параметр микроструктуры $h$ не влияет на напряженное состояние.

На рис. 3 представлено схематическое изображение зоны пластического деформирования материала в зависимости от начального давления. Если $\frac{p_{0}}{Y}>\frac{1}{3(f+\alpha)}$, происходит сжатие материала, если $\frac{p_{0}}{Y}<-\frac{1}{3(f-\alpha)}-$ то растяжение. Таким образом, построенная математическая модель предельного напряженного состояния тонкого вертикального цилиндрического слоя, заполненного сжимаемым сыпучим материалом, находящегося под действием вертикальной нагрузки, позволила оценить зону пластического деформирования материала.

\section{СПИСОК ЛИТЕРАТУРЫ}

1. Быкова М. И., Вервейко Н. Д., Сумеи П. П., Шашкина С. А. Течение и деформирование материалов однородной микроструктуры. - Воронеж: ВГУ, 2010.

2. Быковцев Г. И., Ивлев Д. Д. Теория пластичности. - Владивосток: Дальнаука, 1998.

3. Вервейко Н. Д., Фролов А. Л. Кинематика предельного осесимметричного состояния сыпучих материалов со сцеплением// в кн.: Проблемы механики деформируемых твердых тел и горных пород/ Сб. статей к 75-летию Е. И. Шемякина. - М.: Физматлит, 2006. - С. 107-114.

4. Вервейко Н. Д., Фролова О. А. Влияние характерного размера представительного элемента сыпучей среды на осесимметричное напряженное состояние// Сб. тр. Междунар. конф. «Актуальные проблемы прикладной математики, информатики и механики». - Воронеж, 2011. - С. 106-109.

5. Вялов С. С. Реологические основы механики грунтов. - М.: Высшая школа, 1978.

6. Ивлев Д. Д. Теория идеальной пластичности. - М.: Наука, 1966. 
7. Соколовский В. В. Статика сыпучей среды. - М.: Наука, 1990.

8. Фролов А. Л. Осесимметричное деформирование микрополярных связных сыпучих материалов// Мат. школы-семинара, посв. 70-летию проф. Д. Д. Ивлева. - Воронеж, 2000. - С. 474-480.

9. Фролов А. Л., Фролова О. А. Осесимметричное напряженное состояние связного сыпучего материала с учетом микроструктуры// Вестн. Чуваш. гос. пед. ун-та. Сер. Мех. предел. сост. - 2013. - 1, № 15. - C. 214-221.

10. Eringen A. C. Theory of micropolar fluid// J. Math. Mech. - 1966. — 1, № 16. — P. 1-16.

Фролова Оксана Александровна

Военный учебно-научный центр Военно-воздушных сил

«Военно-воздушная академия им. проф. Н. Е. Жуковского и Ю. А. Гагарина», Воронеж

E-mail: OksanaFrola@yandex.ru 